Orange Journal/Volumen 1 Número 1/ Enero - Junio 2019

DOI: http://dx.doi.org/issn.2710-995X/2019.1.04

\title{
El feminismo: una herramienta para analizar la literatura
}

Feminismo: uma ferramenta para analisar a literatura.

\section{Escrito por: \\ Zahra Hasan Jasim ${ }^{10}$ \\ Akram Hamid Hamzah"11 \\ Muhannad Hadi Abdulameer ${ }^{12}$}

\section{Resumen}

En general, la ideología de la mujer ha variado y cambiado de acuerdo con los conceptos dominantes, y esto ha creado grandes desafíos para las mujeres. La noción de mujer perfecta estaba restringida en relación con las normas sociales en general. Casi no tenía asociación con problemas sociales. Cuando llegó el nuevo siglo, la condición de la mujer se mantuvo igual en los cambios superficiales. Es esta condición la que determina el carácter de la mujer. Se dice que la mujer es prudente, mezquina, falsa y teórica y que no tiene sentido de los hechos y también carece de moralidad. Esto puede ser cierto. Sin embargo, estas variedades de comportamiento de las mujeres no son causadas por sus armonías, ni por la estructura de su cerebro. Por el contrario, están moldeados por su situación. Según Beauvoir, hay ciertas características comunes en los cargos contra la mujer. De hecho, las mujeres nunca han construido una sociedad independiente. Por el contrario, son una parte integral del mundo gobernado por hombres. Durante la segunda guerra mundial, una nueva ola de liberación de mujeres, esto les dio la oportunidad de disolverse en la sociedad y participar en muchas actividades sociales como la fuerza laboral, la educación y la política.

Palabras clave: Feminismo, género, sexo, sumiso, empoderamiento.

\section{Resumen}

Em geral, a ideologia da mulher tem variado e cambiado de acordo com os conceitos dominantes, e os seus grandes desejos são desafiados para as mulheres. A mulher de mulher perfeita estabelece uma relação em relação com as normas sociais em geral. Casi no tenía asociación con problemas sociales. Cuando llegó o novo siglo, a condición de la mujer se mantuvo igual nos cambios superficiales. Esta é a condição que determina o

\footnotetext{
${ }^{10}$ Profesor asistente. Dirección General de la educación/Babil.

${ }^{11}$ Profesor asistente. Dirección General de la educación/Babil.

${ }^{12}$ Profesor adjunto. Universidad de wassit, colegio de Artes, Departamento de traducción.
} 
caráter da mulher. É um dado que a mulher é prudente, mezquina, falsa e teórica e que não tem o sentido dos hechos e também da carece de moralidad. Esto puede ser cierto. $\mathrm{O}$ pecado embargo, estas variedades de comportamento das mulheres não são causadas pelas emoções, mas também pela estructura de seu cerebro. Por contrario, está moldado por sua situación. Según Beauvoir, feno mais características comunais em cargos contra a mulher. De fato, as mulheres nunca construíram uma sociedade independente. Por contrario, filho parte integrante do mundo governado por hombres. Por último, a guerra mundial, uma nova lei de libertação de mulheres, está a ser uma oportunidade de combater a sociedade e a participar em actividades sociais como a vida laboral, a educação e a política.

Palavras-chave: Feminismo, género, sexo, sumiso, empoderamiento

\section{Introducción}

Cada nación tiene su propia mentalidad creativa, artística, literaria y crítica. La cultura y el estándar social de las naciones pueden reflejarse a través de la literatura. El análisis de una literatura de varias maneras necesita aplicar teorías críticas relevantes. Una de las teorías más vibrantes hoy en día para interpretar cualquier pieza de literatura es el punto de vista feminista. El mundo está organizado según el concepto de desequilibrio del patriarcado. Las personas poderosas dominan a los impotentes, los que gobiernan sobre los que no tienen, los hombres establecen el control sobre las mujeres. La mayoría de las sociedades en todo el mundo son patriarcales. En el patriarcado, las mujeres han sido reprimidas, oprimidas y consideradas como un objeto de consumo durante siglos. Los siglos XIX y XX vieron el despertar de la conciencia de la mujer a través de movimientos populares sociopolíticos y literarios. Los autores han estado tratando de retratar una imagen realista de las estructuras sociales y familiares en todo el mundo en sus respectivos textos. Las obras literarias recientes proyectan que las mujeres se rebelen contra su condición secundaria tradicional en la familia y la sociedad. Estas y las obras pasadas deben analizarse desde perspectivas feministas aplicando diversos puntos de vista y teorías dentro del feminismo. La importancia de tener en cuenta el desarrollo de la noción de feminismo, las teorías históricamente famosas sobre el feminismo se plantean para conocer y aplicar a cualquier texto literario.

\section{Planteamiento del problema}

El trabajo tiene como objetivo analizar la condición de las mujeres a través de la literatura y cómo los autores abordaron este problema en sus trabajos. La representación de las mujeres en la literatura ha sido siempre un tema de discusión, y esto es lo que intentamos analizar en este estudio. Como sociedad patriarcal, las mujeres siempre han sido incluidas en el asiento trasero, sin embargo, las mujeres comenzaron a rebelarse y a proclamar la liberación. Por otro lado, hay quienes eligen permanecer pasivos, aceptando su destino sin exigir el cambio.

Considerando este tema, investigamos muchas preguntas relacionadas con la condición de la mujer, la primera y más dominante será: ¿cómo influyó el feminismo en la literatura? A través de muchas obras literarias veremos la emancipación de la mujer. Segundo, ¿cuál 
es la definición general del feminismo? ¿Cómo y en qué circunstancias apareció? ¿Cuáles son sus olas? ¿Cuál es la visión general del feminismo en la literatura? ¿Cómo contribuye la literatura a la emancipación de la mujer? ¿Cómo analizaron, interpretaron y contribuyeron muchos autores y escritores a la emancipación de la mujer?

\section{Alcance y limitación del estudio}

El estudio también sobre la literatura feminista, teniendo en cuenta el importante papel de las escritoras en la emancipación de la mujer. Los escritores usan a las mujeres como personajes en su trabajo para representar la realidad tal como es y presentar historias reales de mujeres a través de los personajes.

\section{Metodología}

Este estudio analiza el estado y las condiciones de las mujeres, sus roles y lo que han pasado para pasar de ser sumisas a ser líderes en literatura, hay fuentes secundarias como libros, internet, artículos y revistas que describen el feminismo.

\section{Revisión de literatura}

Esta parte trata de la revisión de la literatura del estudio actual sobre el feminismo como teoría literaria y se centra en los estudios previos relacionados con el género en la literatura. Esta parte también toma en consideración las principales teorías y teóricos. Además, el estudio rastrea los desarrollos de sexo y género en términos de cultura que determina el papel de la mujer. La emancipación es un tema crucial que fue utilizado por muchos autores y escritores en sus novelas que tenían como objetivo representar las circunstancias difíciles de las mujeres. Luego, la forma en que su papel cambia de ser sumisos a personajes con poder.

\section{Feminismo}

El feminismo en la crítica contemporánea occidental es una teoría prominente. Ha desafiado los supuestos críticos tradicionales. El siglo XX fue testigo de un progreso en esta área. En 'Feminismo' en sí, varias tendencias. Es difícil definir feministas. Aborda cuestiones relacionadas con el estado de las mujeres en la familia y la sociedad, la conciencia femenina, el tratamiento masculino de las mujeres y su opresión. La historia literaria se refiere a los cambios en el lugar y la posición de una mujer. A medida que avanzaba, la sociedad se convirtió en el sistema fundamental en el que la mujer está dominada por el hombre y la sociedad no restringió esta desigualdad.

Naturalmente, la mujer nace libre, pero ciertas normas sociales del hombre la encadenaron. Estas cadenas son obvias en el mundo de la mujer. Hasta el siglo XX, la mayor parte de la literatura británica fue escrita por escritores masculinos, y los personajes femeninos se proyectan desde la perspectiva masculina. Los seudónimos son utilizados por escritoras que asumen hombres como las hermanas Bronte, George Eliot y otros. "Virginia Woolf", en el siglo XX, señaló el tema principal de la mujer cuando trata de alejarse de la dominación de los hombres. 


\section{ORANGE JOURNAL}

La tendencia feminista fue creada por escritoras y críticas femeninas durante el siglo XX. El estado de la mujer normalmente lo decidían los hombres antes de la década de 1960. La conciencia se logró como resultado del sufragio femenino y tenían un rayo de esperanza para resolver los problemas feministas. Los problemas de la mujer no se han resuelto, sin embargo, las mujeres comparten el mundo de los hombres como si tuvieran el derecho de votar. Muchas mujeres se esforzaron por continuar logrando los objetivos que no se lograron.

Las mujeres dieron la bienvenida al nuevo mundo cuando el nuevo movimiento tuvo éxito. La gama de problemas que tuvieron que enfrentar ha cambiado. En Canadá y Estados Unidos, una nueva organización y manifestaciones llegaron a la escena. Estas organizaciones adoptaron la posición de defensa de las mujeres en su sociedad exigiendo justicia e igualdad a los hombres. La gran influencia de escritoras feministas famosas como Germaine Greer, Kate Millet y otras, creó una rebelión contra la opresión y el dominio de las mujeres por parte de los hombres.

El enfoque feminista se considera el único camino para atraer la atención del mundo sobre la injusticia cometida contra las mujeres]. En general, el movimiento feminista creció después del año 1960 '. Aunque, el origen del feminismo se remonta al período anterior en Francia y Holanda 1872, Gran Bretaña 1890.

\section{Auge del feminismo}

Originalmente, el feminismo era una tendencia literaria y luego se convirtió en un movimiento sociopolítico. El feminismo surgió como resultado de estos eventos. La teoría fue occidental en los años sesenta y setenta después de eso ganó su importancia global. Las cuestiones esenciales en el feminismo fueron: ¿qué quiere la mujer? ¿Quién es la verdadera feminista? Estas preguntas son los temas más dominantes en el feminismo. Carmen Vásquez dice que "las feministas no pueden ponerse de acuerdo sobre qué feminista" y afirma además que "hay tantas definiciones como feministas". La crítica del feminismo moderno presenta una amplia gama de situaciones femeninas: a) se utiliza el tipo de lenguaje para escribir sobre la mujer b) el psicoanálisis del valor femenino c) la función del feminismo. Showalter mencionó los principales intereses de los conceptos críticos tradicionales como la caracterización, los motivos y el tema. Algunos críticos como Derrida creían que el mundo real no se presenta en los textos literarios.

\section{Olas del feminismo}

La lucha por la igualdad ha continuado durante más de cien años. La historia de esta lucha puede considerarse como olas. Diferentes aspectos de los problemas feministas abordaban cada ola con diferentes objetivos.

\section{Primera ola de feminismo}

La actividad de finales del siglo XIX y del siglo XXI se refiere a la primera ola de feminismo en el mundo. Los países que presenciaron el primer período de feminismo son Gran Bretaña, Países Bajos, Canadá y Estados Unidos. Los principales problemas de la 
primera ola de feminismo son la posición de la mujer y el movimiento del sufragio y se concentra en cuestiones legales como la educación y las condiciones de trabajo. En 1906, un artículo en un periódico británico utilizó la palabra "sufragio" para describir la lucha de las mujeres por los derechos de voto. Varios puntos de vista de hombres y mujeres están involucrados en el movimiento sufragista. En 1903 en Gran Bretaña, se formó la primera Unión de mujeres y esta unión intentó trabajar en la liberación de la mujer. Aunque hubo una controversia sobre el lugar de la mujer, el movimiento intentaba obtener la posición correcta en la sociedad.

Los rasgos naturales de la mujer hicieron que los activistas sintieran que las mujeres son amables y están más preocupadas por los miembros semanales de la sociedad. La votación de las mujeres tendría un efecto civilizador en la política. Otros pidieron igualdad de hombres y mujeres en todo.

\section{Segunda ola de feminismo}

A mediados de la década de 19 y mediados de la década de 1960, comenzó la segunda ola de liberación de las mujeres en los Estados Unidos y continuó hasta mediados de la década de 1980. Se convirtió en un patrón mundial que fue dinámico en partes de Asia, por ejemplo, Turquía e Israel, Europa y donde comenzó durante la década de 1980 y, además, comenzó en diferentes ocasiones en diferentes naciones. La ola principal de liberación de las mujeres se centró en el equilibrio de la orientación sexual, pero la segunda ola de derechos de las mujeres tuvo una visión más amplia que antes. Toma en consideración temas como la violación marital. Los años 60 vieron el comienzo de la segunda ola de liberación de las mujeres en los Estados Unidos y continuaron hasta mediados de los años ochenta. Se convirtió en un patrón mundial que fue dinámico en partes de Asia, por ejemplo, Turquía e Israel, Europa y donde comenzó durante la década de 1980 y, además, comenzó en diferentes ocasiones en diferentes naciones. Los objetivos principales de la ola fueron la liberación de las mujeres y se centraron en el equilibrio de la orientación sexual, sin embargo, la segunda ola de derechos de las mujeres tuvo una visión más amplia que antes. Toma en consideración cuestiones como la violación marital. se centró en ciertos problemas de desigualdad legal, incluida la discriminación laboral, la sexualidad y los problemas familiares. La acción nacional sobre el comité de mujeres se formó cuando la Comisión Real de mujeres canadienses tiene razón. La comisión se centró en las condiciones femeninas durante los años 1970 y 1980. Esto creó una gran influencia en los escritos literarios. La lucha y los derechos de las mujeres se reflejaron altamente en las producciones de los autores.

\section{Tercera ola de feminismo}

En 1992, Rebecca Walker utiliza el término feminismo de la tercera ola en su ensayo. pero, el desarrollo comenzó a mediados de la década de 1980. Surgió debido a las decepciones de la segunda ola. También fue una reacción al discernimiento de que las mujeres son de "numerosos tonos, etnias, nacionalidades, religiones y fundamentos sociales". El movimiento de la liberación de la segunda ola de mujeres se produjo con un gran número de los derechos legítimos e institucionales extendidos a las damas. Además, las activistas de mujeres de la tercera ola confiaron en que debería haber más cambios en 


\section{ORANGE JOURNAL}

generalizaciones, representaciones de los medios y lenguaje para caracterizar a las mujeres.

El sistema de creencias de la tercera ola se centra en una traducción más postestructuralista del sexo y la sexualidad. Los problemas importantes de este desarrollo fueron la raza y la clase. La liberación de la tercera ola femenina fue una mezcla de las dos oleadas anteriores. La ola primaria identificada con los privilegios legítimos de las damas y para eso, comenzó el desarrollo del sufragio. Para decirlo claramente, fue el círculo principal donde la mujer dejó su propio espacio que es la casa. La segunda ola implica el segundo círculo donde la mujer obtiene sus derechos sociales y legítimos en los entornos de trabajo, y la tercera ola presenta el tercer círculo donde encuentran sus propios personajes en la arena pública. La tercera ola del feminismo ofreció certeza a las activistas en el campo.

Las tres olas se centran en el tema central del feminismo. Hay preguntas sobre cómo la mujer puede salir de su propio círculo creado por la sociedad. Las tres olas proporcionaron la respuesta a esta pregunta.

\section{Patriarcado: una fuente de dominación femenina}

La mayoría de las comunidades de todo el mundo son patriarcales. Patriarcado es un término que indica la dominación masculina sobre la familia dentro del contexto social. Las mujeres siempre han sufrido el control, la presión y la represión masculinos dentro y fuera de la familia. A pesar de estar empoderadas y dominadas por los hombres, las mujeres siempre luchan por obtener sus derechos y luchan por mantenerse. El deseo de cada mujer individual de superar el patriarcado se debe a su deseo de ser independiente. El patriarcado también se define a la luz de la institucionalización del dominio de los hombres sobre las mujeres y los niños dentro del contexto familiar. Este dominio a menudo se extiende para exceder a la familia hasta los límites sociales. La opresión y la subyugación son las consecuencias del patriarcado porque la mujer siempre se presenta como sujeto de los hombres. Hombres, en las unidades sociales más importantes; La familia, posee poder. Esta retención de poder da como resultado que los hombres traten a las esposas e hijos como sus propiedades privadas. La familia de base capitalista se considera la unidad de poder más pequeña dentro de la estructura social. Esta consideración, que se atribuye a los ángeles de Frederick, ha permitido a los hombres practicar formas similares de poder fuera de la familia.

\section{Autorrealización}

Los seres humanos, en última instancia, tienen como objetivo obtener la autorrealización para lograr una alegría perpetua, a través de la independencia y la liberación de la esclavitud. Esa verdadera alegría resulta de la autorrealización. La autorrealización significa el cumplimiento de las propias capacidades latentes del individuo. La conciencia de los poderes, las capacidades y el respeto de sí mismo del individuo se traduce en la autorrealización. Brevemente, la autorrealización se considera un desarrollo humano, que es la parte superior de todos los esfuerzos morales. Intentar darse cuenta de sí mismo, el poder, la autoestima y la alegría dentro de la familia y las estructuras sociales nunca han 
estado entre los intereses de las mujeres hasta hace poco. Las obras literarias que se ocupan de las mujeres durante el período posmoderno intentan planificar para las protagonistas femeninas, ya que las mujeres siempre tratan de lograr la autorrealización y la identidad propia. Por lo tanto, es motivador abordar cómo varios autores planean para sus protagonistas femeninas en un intento de aclarar cómo las mujeres hacen lo mejor para lograr la autorrealización.

\section{Sexo y género}

La distinción entre hombres y mujeres a la luz de las variaciones biológicas, físicas y genéticas se marca a través del sexo y el género. Los roles de género se establecen por convención. Hay otros factores sociales y económicos además de los factores políticos y culturales que determinan los roles de género. Por lo tanto, el sexo es un concepto natural, mientras que el género es un concepto cultural. "Sexo" indica una variación biológica que está relacionada con los cromosomas, los genitales y las funciones reproductivas de los seres humanos, que definen a cada individuo como una "mujer" o un "hombre". Pero, prácticamente, los seres humanos están interesados en cómo camina un individuo, los cortes de pelo del individuo y la ropa del individuo. Además, hay otras fisionomías físicas y conductuales que no están biológicamente identificadas. El género de la persona diferencia a los hombres de las mujeres. El género determina cómo los seres humanos están socialmente construidos para comportarse y practicarse como "hombres" o "mujeres". En el patriarcado, los hombres son anteriores a las mujeres. Las opiniones de "Simon De Beauvoir" sobre las situaciones y la naturaleza del tratamiento de las mujeres son radicales. La canción fue controvertida en su libro inspirador "The Second Sex '(1949)". El libro Reconstruyó la forma de pensar de los autores y críticos sobre las mujeres en el nivel secundario.

\section{Cuerpos}

El pensamiento occidental asocia históricamente el "cuerpo" con las mujeres y la "mente" con los hombres. La dualidad natural de la relación "mente / cuerpo" debido a las primeras filosofías de "Aristóteles, Hegel y Descartes" es elaborada por la filósofa feminista moderna "Susan Bordo" en sus escritos en un intento de revelar cuán distintivos son los binarios; "Espíritu / materia y actividad masculina / pasividad femenina" interactúan para fortalecer la fisionomía y categorización de género. La noción de asociar "cuerpo" con "mujeres" ha llevado históricamente a la justificación de considerar a las mujeres como "propiedad, objeto y mercancía intercambiable" para los hombres. Los principios cambiantes de "moda, dieta, programas de ejercicio, cirugía estética, maternidad, etc." han resultado históricamente en objetivar el cuerpo de la hembra.

El hombre es visto como un agente moral que se encarga de trabajar o ir a luchar en guerras sangrientas. La "raza y clase de una mujer" determina la forma en que se trata su cuerpo. A la luz de estos dos factores, el cuerpo de la hembra es visto como una decoración y protección; Los cuerpos de las hembras en clases trabajadoras o con hembras de colores. Hay un feminismo de segunda ola que defiende los "derechos y elección reproductivos, la salud de las mujeres y los derechos de las lesbianas". Todos estos asuntos también están asociados con la noción de "cuerpos". 


\section{ORANGE JOURNAL}

\section{Subjetividad y mujeres}

Subjetividad es el término que indica acción o discurso producido por el individuo o "yo": el sujeto. Indica el sujeto y su "perspectiva, sentimientos, creencias y deseos". Históricamente, las mujeres siempre han sido los sujetos de su vida. Las mujeres han tenido alguna oportunidad de discutir diferentes métodos de independencia y tomar decisiones. Sin embargo, estos métodos no denotan libertad total. Al menos, permiten que las mujeres demuestren sus necesidades, deseos y desafían las condiciones dadas a las mujeres como su destino. La subjetividad tiene varios significados. Primero, toma en consideración la capacidad de imaginar, pensar y tomar decisiones. Segundo, indica la liberación de las mujeres; "Trabajar fuera del hogar, usar anticonceptivos y educarse, la oportunidad de tener diferentes formas de relación y usar lo que quieran". La subjetividad involucra todas estas formas. Pero, debe notarse que todos los métodos de liberación e independencia pueden no ayudar a las mujeres a superar los "estereotipos patriarcales". A pesar del hecho de que no se puede lograr un verdadero sentido de libertad, pero, al menos, a las mujeres se les da cierto alcance para "tomar decisiones sobre cosas muy pequeñas".

\section{Gynocriticism}

El término "Gynocritics" ha sido acuñado por la feminista estadounidense "Elaine Showalter". Al acuñar el término, esperaba mejorar los modelos novedosos basados en la experiencia femenina en lugar de la devoción ciega a los modelos masculinos. "Gynocriticism" indica el estudio literario de "mujeres como escritoras". Explora y registra la creatividad femenina, ya que es una práctica crítica. Comprender la escritura de las mujeres como una parte esencial de la realidad femenina se logra a través del "Ginecitismo". A través del "Gynocriticism", "Elaine Showalter" quiere formar una tradición literaria a través de la cual las mujeres se expresen sin combinar autores masculinos. Ella siente que la crítica feminista todavía se guía por suposiciones masculinas. El "ginecritismo" es una nueva etapa del "autodescubrimiento" de las mujeres.

\section{Tipos de feminism}

Después de los años sesenta, hubo varios tipos de "feminismos" como: "Feminismo radical, feminismo socialista, feminismo liberal, feminismo marxista, feminismo negro, feminismo lésbico, feminismo burgués, feminismo materialista, etc.". "Feminismo" es un término exigido por las "mujeres blancas de clase alta" para oponerse al nuevo término "feminismo" que representa a toda la comunidad de mujeres. El desarrollo del "feminismo" se estudia de manera prominente a la luz de tres orientaciones feministas distintas como se mencionó anteriormente.

\section{Mujer nueva}

La "Mujer Nueva" es esa mujer que se ha vuelto consciente de su propia identidad, espacio en la sociedad, espacio en la familia y espacio en su propia vida. Lo que las mujeres sufrieron en el pasado ha fortalecido su psique. Pero la maternidad los ha 
debilitado, ya que los ha hecho dependientes. Al darse cuenta de la obligación de obtener un grado considerable de perfección y deshacerse de la impresión sobre el futuro, la educación ha resultado en la expansión de las ideas y las ideas de las mujeres. poder mental. Habiendo surgido durante la década de 1890 en América del Norte y Europa, el término "Mujer Nueva" llegó a representar los ideales de las feministas. "Nueva mujer" fue ideada por primera vez por la escritora "Sarah Grand" en su artículo "El nuevo aspecto de la pregunta de la mujer (1894)". Fue promovido por el escritor británicoestadounidense "Henry James" para describir la evolución en el número de "mujeres feministas, educadas e independientes orientadas a la carrera en Europa y los Estados Unidos". "Nueva mujer" siempre se refiere a las mujeres que practican el control sobre sus propias "vidas personales, sociales o económicas". Las características importantes de la "Nueva Mujer" son:

- Ella se da cuenta de sus emociones y sentimientos libremente.

- Ella recibe una cantidad adecuada de educación para usar a sus conocidos de manera inteligente.

- Ella gana dinero y se vuelve financieramente independiente.

- Ella decide sobre su matrimonio y descendencia.

- Es capaz de confrontar convenciones y normas sociales.

- Ella confía en sí misma y confía en que puede enfrentar los cambios emocionales y psicológicos que la rodean.

Estas características ayudan a comprender el carácter fundamental de "La mujer comestible" que la escritora "Atwood" pone en práctica entre las mujeres canadienses de la época.

\section{Empoderamiento}

Una enorme discusión sobre el empoderamiento de la mujer en los campos sociopolíticos Ha habido una tremenda discusión sobre el empoderamiento de las mujeres en el ámbito sociopolítico. El término empoderamiento con su asociación con el feminismo se refiere a despertar la aplicación espiritual, política, social o económica de comunidades y personas también. El empoderamiento podría representar las capacidades de las feministas para involucrarse en diferentes aspectos sociales, como su propio poder de toma de decisiones o tomar decisiones adecuadas mediante el acceso a los recursos de información.

El empoderamiento se refiere al proceso que intenta evitar las fuerzas que marginan a las mujeres. El término poder no pretende lograr una forma de dominación de las mujeres sobre los hombres, sino que exige los derechos que hacen de las mujeres un miembro activo del poder social. El empoderamiento se basa en dos cosas. Primero, debe haber un poder que pueda traer un cambio y allanar el camino hacia el empoderamiento; en segundo lugar, esencialmente adquiere el poder de pensar y comportarse libremente y también de presentar el potencial de ser un miembro activo de la sociedad.

Es esencial reclutar a las famosas feministas que fortalecieron y enriquecieron el feminismo. Sus contribuciones junto con otras feministas marginales son de gran valor. 


\section{ORANGE JOURNAL}

Una de las escritoras prominentes del siglo XX es Virginia Woolf (1882-1941). ella escribió muchas novelas famosas como Mrs. Dalloway, Orlando, To the Lighthouse y otras obras no ficticias como "A Room of One's (1929)" y Three Guineas (1938) "que trata sobre el dominio de los hombres sobre las mujeres. Wolf a menudo se cita para: "Una mujer debe tener dinero y una habitación propia para escribir ficción".

La escritora feminista francesa Simone de Beauvoir (1908-1986) escribió "El segundo sexo (1949)". Abogó por los problemas esenciales y las experiencias de las mujeres. El análisis de Beauvoir de los motivos de inferioridad de la mujer al hombre y por qué se realiza como "otra" en una sociedad y recibe el tratamiento de los hombres en la sociedad como segundo sexo.

Según sus mujeres, ella puede traer el cambio en sus condiciones sociales. Luce Irigaray (1930-), la famosa feminista francesa en sus libros traducidos "Speculum, of the Other Woman" (1985). Ella critica el punto de vista centrado en el falo en la teoría del psicoanálisis.

En "El sexo que no es uno" (1977), intenta presentar la ausencia de un papel femenino en la sociedad occidental. otra feminista francesa es Julia Kristeva (1941-) quien presentó sus mejores puntos de vista sobre el feminismo en su ensayo "Tiempo de las mujeres en nuevas enfermedades del alma" (1978). Ella discutió las tres fases del feminismo; La falta de igualdad y las diferencias sexuales la hacen rechazar la primera fase. Ella critica el rechazo de Beauvoir del concepto de maternidad y quiere descubrir un nuevo discurso sobre la maternidad.

Helen Cixous (1937-) es considerada como una de "Las madres de la teoría feminista postestructuralista". En la década de 1970, ella trató las relaciones entre el lenguaje y la sexualidad en su escritura. Considera la forma en que las personas tienen comunicación social a través de la sexualidad. En 1975, escribió su ensayo más famoso "La risa de la medusa". Presenta un modelo de pensamiento y escritura sobre literatura feminista y femenina. Llamó al término "La ecriture femenino", es decir, "escritura femenina".

La conocida crítica feminista estadounidense Mary Ellmann (1921-1989) "Thinking about Women" (1968) discute la presentación de la mujer en la literatura británica y estadounidense, mostrando analogía sexual y mujeres luchadoras del texto y críticas contrastantes de hombres y mujeres. autores.

Necesidad de desarrollar un modelo para el análisis feminista de textos literarios.

Para hacer un análisis de cualquier obra literaria es necesario tener un modelo teórico basado en un marco teórico. Los autores adoptan diferentes posturas para delinear con mujeres y temas relacionados con las mujeres. Un investigador puede desarrollar un modelo apropiado que se ajuste para analizar el trabajo en cuestión. Los autores exponen la orientación del mundo masculino en las relaciones, en el lugar de trabajo, en el hogar, en las organizaciones, en la sociedad, oprime a las mujeres. Las protagonistas femeninas y muchos otros personajes femeninos exhibieron varios modelos de comportamiento femenino en las obras. Algunas mujeres aceptan la realidad, mientras que la otra se rebela contra el sistema y las costumbres para trazar un nuevo lugar para ellas. Los hombres han 
estado tratando a las mujeres como un objeto y, por lo tanto, están perturbadas y se encuentran en un dilema. Todos estos temas en los trabajos relevantes pueden discutirse utilizando un paradigma teórico concreto. Diferentes escritoras feministas tienen puntos de vista aplicables al analizar novelas feministas. Los puntos de vista de Second Sex of Simone de Beauvoir son aplicables a lo que los personajes femeninos en general experimentaron. Como Mary Ellmann, Elaine Showalter y Betty Friedan han sugerido que una nueva mujer intenta trazar su propia identidad. Por lo tanto, las teorías críticas se han vuelto fructíferas para analizar los personajes femeninos.

\section{Conclusión}

El feminismo y los problemas de las mujeres son prominentes entre los investigadores y críticos tardíos. Se ve que la estructura hipotética feminista es fundamental para la investigación de temas femeninos en cualquier obra literaria. Posteriormente, las discusiones teóricas sobre modelos ideales en los derechos de la mujer son necesarias para lograr una mejor realización y análisis de cualquier obra de arte relacionada con las feministas. El presente documento se centra en uno de los problemas identificados con la liberación de las mujeres y el desarrollo de varias etapas por las que han pasado los derechos de las mujeres, los estudiosos importantes y sus perspectivas. Los derechos de la mujer se centran en el predominio masculino que es sinónimo de sociedad centrada en el hombre. Este predominio y los problemas identificados con la mujer libre de las servidumbres de los hombres y las estructuras socio-familiares han sido investigados por varias críticas feministas y han exhibido sus perspectivas en artículos básicos relativos y libros que representan la teoría feminista. La investigación de los problemas identificó que la liberación femenina y el desarrollo feminista se han establecido en el período posterior a la Segunda Guerra Mundial. Las teorías del feminismo ciertamente ayudarían a los críticos e investigadores a hacer un mejor análisis del trabajo artístico actual o pasado

\section{Referencias}

Balachandranana, K. Ensayos sobre literatura canadiense. Bareilly: Prakash Books Depot, 2001.

Barry, Peter. "Teoría inicial: una introducción a la teoría literaria y cultural". Tercera edición, Nueva Delhi: Viva Books.

Beauvoir, Simone De. "Mujeres y creatividad", pensamiento feminista francés: A Reader, ed. Toril Moi. Basil Blackwell, 1987, 19.

Cixous, Helen. "La risa de la Medusa" trans. Keith Cohen y Paula Cohen, Signs, 1.4 Summer 1976, 875-93.

De Beauvoir, Simone. El segundo sexo (1949). Traducido por H. M. Parshely, Nueva York: Vintage Books.

Dorothy Kaufmann McCall. Simone De Beauvoir, El segundo sexo, y Jean Paul Sartre. Londres: Rutledge, 1979, p.210

Mitchell, Julieta. El complejo de Edipo Obsolescente, y el declive del Patriarcado Journal of Soc. Y Psy. Sci. 2007 volumen 1 (1): 17-

Citado por N. Krishnaswamy.et.al. Teoría literaria contemporánea. Nueva Delhi: Macmillan, 2001, p.73 


\section{ORANGE JOURNAL}

Shukla, Bhaskar. Feminismo y postfeminismo: el contexto de las poetas de la India moderna Escritura en inglés. Nueva Delhi: Sarup and Sons, 2004.p.30

Singh, Sushila. Feminismo y ficción reciente en inglés. Nueva Delhi: Prestige Books, 1991. 